\title{
Cost Comparison and Complication Rate of Lisfranc Injuries Treated with Open Reduction Internal Fixation versus Primary Arthrodesis
}

\author{
Brandon Barnds, MD1, William Tucker, MD, Brandon L. Morris, MD², John Paul Schroeppel, MD³, Scott M. Mullen, MD, \\ Armin Tarakemeh, BA ${ }^{1}$, Bryan George Vopat, MD $^{4}$
}

${ }^{1}$ University of Kansas Medical Center, Kansas City, KS, USA, ${ }^{2}$ Univ. of Kansas Medical Center Dept. of Orthopedic Surgery, Kansas City, KS, USA, ${ }^{3}$ Kansas University Orthopedics and Sports Medicine, Kansas City, KS, USA, ${ }^{4}$ University of Kansas, Overland Park, KS, USA.

Objectives: Controversy exists regarding optimal primary management of Lisfranc injuries. Whether open reduction internal fixation or primary arthrodesis is superior remains unknown. Our retrospective study uses a private payer database to compare cost, complication rate, and hardware removal rate in Lisfranc injuries treated with primary open reduction internal fixation or primary arthrodesis.

Methods: Utilizing data mining software created by a private organization, a national insurance database of approximately 23.5 million orthopedic patients was retrospectively queried for subjects who were diagnosed with a Lisfranc injury from 2007-2016 based on international classification of diseases (ICD) codes for tarsometatarsal (TMT) dislocation (PearlDiver, Colorado Springs, CO). Patients with TMT dislocations then progressed on to either non-operative treatment, open reduction internal fixation, or primary arthrodesis. Associated treatment costs based on diagnosis codes were followed after initial diagnosis and t-tests were used to determine statistical significance. Subgroups were then created based on having at least one complication ICD or current procedural terminology (CPT) code after the beginning of treatment, which included: hemorrhage, infection, nonunion, malunion, thromboembolism, wound and hardware complications, or amputation. Additionally, patients undergoing implant removal were identified by CPT code for removal of hardware performed after the index procedure. Complication and hardware removal rates were compared with chi-square test.

Results: 2205 subjects with a diagnosis of Lisfranc injury were identified in the database. 1248 patients underwent non-operative management, 670 underwent open reduction internal fixation, and 212 underwent primary arthrodesis. The average cost of care associated with primary arthrodesis was greater $(\$ 5,005.82)$ than for open reduction internal fixation $(\$ 3,961.97, P=0.045)$. The overall complication rate was $23.1 \%(155 / 670)$ for open reduction internal fixation and $30.2 \%(64 / 212)$ for primary arthrodesis $(P=0.04)$. Rates of hardware removal independent of complications were $43.6 \%$ (292/670) for open reduction internal fixation and $18.4 \%(39 / 212)$ for arthrodesis $(P<0.001)$. Furthermore, $2.5 \%(17 / 670)$ patients in the open reduction internal fixation group progressed to arthrodesis at a mean of 308 days, average cost of care associated with this group of patients was $\$ 9,505.12$. Conclusion: Primary arthrodesis for the management of acute Lisfranc injuries is both significantly more expensive and has a higher complication rate than open reduction internal fixation. Open reduction internal fixation demonstrated a low rate of progression to arthrodesis, although there was a high rate of hardware removal, which may represent a planned second procedure in the management of a substantial number of patients treated with open reduction internal fixation.

The Orthopaedic Journal of Sports Medicine, 6(7)(suppl 4)

DOI: $10.1177 / 2325967118 S 00122$

CThe Author(s) 2018

\footnotetext{
This open-access article is published and distributed under the Creative Commons Attribution - NonCommercial - No Derivatives License (http://creativecommons.org/licenses/by-nc-nd/4.0/), which permits the noncommercial use, distribution, and reproduction of the article in any medium, provided the original author and source are credited. You may not alter, transform, or build upon this article without the permission of the Author(s). For article reuse guidelines, please visit SAGE's website at http://www.sagepub.com/journals-permissions.
} 